\title{
Transcriptomic alterations in malignant pleural mesothelioma cells in response to long-term treatment with bullfrog sialic acid-binding lectin
}

\author{
TAKEO TATSUTA, ARISU NAKASATO, SHIGEKI SUGAWARA and MASAHIRO HOSONO \\ Division of Cell Recognition, Institute of Molecular Biomembrane and Glycobiology, \\ Tohoku Medical and Pharmaceutical University, Sendai, Miyagi 981-8558, Japan
}

Received February 3, 2021; Accepted March 29, 2021

DOI: $10.3892 / \mathrm{mmr} .2021 .12106$

\begin{abstract}
Malignant pleural mesothelioma (MPM) is a universally lethal type of cancer that is increasing in incidence worldwide; therefore, the development of new drugs for MPM is an urgent task. Bullfrog sialic acid-binding lectin (cSBL) is a multifunctional protein that has carbohydrate-binding and ribonuclease activities. cSBL exerts marked antitumor activity against numerous types of cancer cells, with low toxicity to normal cells. Although in vitro and in vivo studies revealed that cSBL was effective against MPM, the mechanism by which cSBL exerts antitumor effects is not fully understood. To further understand the mechanism of action of cSBL, the present study aimed to identify the key molecules whose expression was affected by cSBL. The present study established cSBL-resistant MPM cells. Microarray analyses revealed that there were significant pleiotropic changes in the expression profiles of several genes, including multiple genes involved in metabolic pathways in cSBL-resistant cells. Furthermore, the expression of some members of the aldo-keto
\end{abstract}

Correspondence to: Dr Masahiro Hosono, Division of Cell Recognition, Institute of Molecular Biomembrane and Glycobiology, Tohoku Medical and Pharmaceutical University, 4-4-1 Komatsushima, Aobaku, Sendai, Miyagi 981-8558, Japan E-mail:mhosono@tohoku-mpu.ac.jp

Abbreviations: RNase, ribonuclease; ONC, onconase; cSBL, bullfrog sialic acid-binding lectin; DEG, differentially expressed gene; ATF3, activating transcription factor 3; cSR, cSBL-resistant; DOX, doxorubicin; AKR, aldo-keto reductase; KEGG, Kyoto Encyclopedia of Genes and Genomes; RT-qPCR, reverse transcription quantitative polymerase chain reaction; PPI, protein-protein interaction; SLC47A2, solute carrier family 47 member 2; CBR, carbonyl reductase; GAPDH, glyceraldehyde-3-phosphate dehydrogenase; RR, resistance rate; $\mathrm{RIN}^{\mathrm{e}}$, RNA integrity number equivalent; GO, Gene Ontology

Key words: antitumor drug, sialic acid-binding lectin, cytotoxic ribonucleases, microarray profiling, metabolism of cancer cells, aldo-keto reductase reductase family was revealed to be markedly downregulated in these cells. Among these, it was particularly interesting that $\mathrm{CSBL}$ action reduced the level of AKR1B10, which has been reported as a biomarker candidate for MPM prognosis. These findings revealed novel aspects of the effect of cSBL, which may contribute to the development of new therapeutic strategies for MPM.

\section{Introduction}

Malignant pleural mesothelioma (MPM) is an aggressive cancer of the mesothelial cells lining the pleural surface of the chest wall and lung (1). The main cause of carcinogenesis of this disease is thought to be exposure to an environmental carcinogen, asbestos (2); furthermore, the involvement of SV40 and exposure to radiation have been suggested as cofactors $(3,4)$. The MPM patient prognosis is very poor and available therapies have still a limited impact on MPM progression (5). Until recently, antifolate and platinum combination chemotherapy was the only established treatment $(6,7)$. These days, the development of immune-checkpoint inhibitors has contributed to the improvement of MPM treatment. The data from the phase III trial (NCT02899299) designed to evaluate nivolumab plus ipilimumab compared with conventional chemotherapy (pemetrexed and cisplatin or carboplatin) showed a statistically significant overall survival (OS) benefit in patients with previously untreated, unresectable MPM, and, only recently, the FDA has finally approved nivolumab plus ipilimumab for previously untreated unresectable MPM (8). Although the nivolumab and ipilimumab combination has extended the OS (a median OS of 18.1 months compared to 14.1 months for platinum-based standard of chemotherapy) (8), the prognosis is still poor, and research such as identification of new biomarkers in invasive mesothelioma (9), and search for new drugs for MPM (10) have been actively conducted for further improvement of MPM care. In particular, the development of drugs through different mechanisms of action will continue to be required in the future.

A major focus in cancer research is the development of new therapeutic agents that induce cell death in malignant neoplasms but do not increase inflammation or have significant side effects in normal tissue. Cytotoxic ribonucleases 
(RNases) are a new field of anticancer drug candidates that target RNA and several cytotoxic RNases have been reported to have antitumor effects $(11,12)$. The vertebrate-secreted RNase superfamily, also called the RNase A superfamily, includes several cytotoxic RNases, such as onconase (ONC) from Rana pipience (13) and a variant of human pancreatic ribonuclease carrying a nuclear localization signal (PE5) (14). Unlike clinically used chemotherapeutic drugs that target DNA synthesis and transcription, cytotoxic RNases are believed to be non-mutagenic because they target RNA functions, such as RNA translation or gene regulation (15).

Sialic acid-binding lectin from Rana catesbeiana (cSBL), also known as RC-RNase, is a multifunctional protein that binds carbohydrates and has a ribonuclease activity (16-18). It was originally identified as a lectin that recognizes sialic acid-containing complexes (17), and protein sequence analysis revealed that it belonged to the vertebrate-secreted RNase superfamily (19). It has previously been identified that cSBL has remarkable antitumor activity against many types of cancer cells and low toxicity in normal cells (20-25). This effect was observed in not only in vitro experiments but also in vivo studies $(18,20,26)$. Our own recent studies revealed that cSBL induced apoptosis in cancer cells via the intrinsic pathway $(27,28)$, and that the RNase activity of cSBL was essential for its antitumor effect (29). The effectiveness of cSBL has also been studied for in MPM. We reported that although cSBL had very low cytotoxicity in the normal pleural mesothelial cell line Met5A, it efficiently reduced the viability of MPM cells including H28, Meso-1, Meso-4, H2452 and MSTO cells $(30,31)$. We found that pemetrexed + cSBL exhibited a strong synergistic effect that was even superior to the standard regimen of pemetrexed + cisplatin (31). Furthermore, in vivo study revealed that $\mathrm{cSBL}$ showed a significant tumor growth inhibitory effect in multiple MPM xenograft models without any adverse effects, even under conditions where previously established pemetrexed administration had little or no effect (26). However, the antitumor mechanism of cSBL is still unclear, especially when the response of cancer cells to cSBL application is concerned.

Despite the potential of RNases in cancer treatment, few studies have identified genes whose expression was altered by cytotoxic RNases. This may be because the RNA extracted from cytotoxic RNase-treated cells is likely to be degraded by the RNA-catabolizing action of the RNase. Therefore, it is technically difficult to assess differentially expressed genes (DEGs) in cytotoxic RNase-treated cells. In recent years, some remarkable research breakthroughs have been made in studies using microarray analysis. Previous studies using microarray technology have been able to determine that ONC caused upregulation of activating transcription factor 3 (ATF3), which was important for its antitumor effect of ONC $(32,33)$, and that PE5 caused pleiotropic effects, including gene expression changes mainly related to metabolism (34). These studies pioneered the study of gene expression after treatment with cytotoxic RNases. However, these findings were reported only in conditions in which there was little RNA degradation, that is, there was very little antitumor effect. Moreover, no gene expression studies have involved cSBL.

To further understand the antitumor effects of cSBL, we treated cSBL-sensitive MPM cells with cSBL to establish
cSBL-resistant (cSR) cells. Then, microarray analysis was performed to identify significantly altered genes in the cSBL-sensitive and cSR cell lines.

\section{Materials and methods}

Reagents. cSBL was isolated from acetone-dried powder of unfertilized bullfrog body-cavity eggs using sequential chromatography with Sephadex G75, DEAE-cellulose, hydroxyapatite, and SP-Sepharose (Cytiva), as previously described (17). For the preparation of ONC, ONC cDNA was cloned into the pET-11d plasmid (Merck KGaA) in conjunction with the pelB sequence. BL21 (DE3) pLysS cells (Promega) were transformed with the plasmid, and its expression was induced by adding isopropyl $\beta$-D-1-thiogalactopyranoside $(0.2 \mathrm{mM})$ at $34^{\circ} \mathrm{C}$ for $72 \mathrm{~h}$. ONC recombinant protein was purified from the culture liquid by sequential chromatography with Sephadex G75, DEAE-cellulose, hydroxyapatite, and SP-Sepharose. Doxorubicin (DOX) was purchased from Sigma-Aldrich. The anti-caspase-3 antibody (cat. no. \#9662), peroxidase-conjugated anti-mouse $\operatorname{IgG}$ and anti-rabbit $\mathrm{IgG}$ antibodies (cat. no. \#7074 and \#7076, respectively) were purchased from Cell Signaling Technology. The anti-aldo-keto reductase (AKR) 1B10 antibody (cat. no. ab96417) was purchased from Abcam (Cambridge, UK) The anti- $\beta$-actin antibody (clone AC-74, cat. no. A2228) was purchased from Sigma-Aldrich.

Establishment of cSBL-resistant cell lines. H28 cells were purchased from the American Type Culture Collection. For the establishment of cSR cell lines, cells were cultured with complete medium containing stepwise increasing concentrations of cSBL. The starting concentration was $0.01 \mu \mathrm{M}$ in which $\mathrm{H} 28$ cells could manage to survive, and the final concentration was set as $0.5 \mu \mathrm{M}$, which is 50 times higher than the initial concentration. The cells were passaged approximately 50 times to reach the final concentration. These resistant cells were proven to be stably resistant even after at least 10 passages in drug-free complete medium. After that, cells were cloned using limiting dilution. We confirmed five resistant clones, which were designated cSR-A1, -A2, -B1, -B2 and -C1. To ensure that these clones were permanent and stable, they were cultured for five passages in the presence of $0.5 \mu \mathrm{M}$ cSBL. For the analyses described here, cultures of less than 20 passages after the cloning were used. All cell lines were cultured in RPMI 1640 medium (Nissui) supplemented with $10 \%$ fetal bovine serum, penicillin-G (100 U/ml), and streptomycin (100 g/ml) (Thermo Fisher Scientific, Inc.). Cells were maintained in a $5 \% \mathrm{CO}_{2}$ incubator at $37^{\circ} \mathrm{C}$ under humidified conditions. Cell morphology was observed using an IX71 microscope (Olympus).

Colony formation assay. Cells were seeded in 6-well plates (Corning, cat. no. 353046, 1×10 $0^{3}$ cells per well). After $24 \mathrm{~h}$, cells were treated with medium alone or with $\mathrm{cSBL}(1,5,10$, 25 , or $50 \mathrm{nM}$ ) for 12 days. Then, cells were fixed with paraformaldehyde for $15 \mathrm{~min}$ and stained with crystal violet for 10 min. Colonies were photographed by Gel Doc XR system (Bio-Rad Laboratories, Inc.) and counted using Quantity One software (Bio-Rad Laboratories, Inc.). Each experiment was performed in triplicate. 
WST-8 assay. The WST-8 assay was performed to determine the cell viability. Cells $\left(5 \times 10^{4}\right.$ cells $\left./ \mathrm{ml}\right)$ cultured in $96-$ well plates (Corning, cat. no. 353072, $100 \mu \mathrm{l} /$ well) were treated with cSBL, ONC, or DOX at the indicated concentrations for $72 \mathrm{~h}$. Then, the cells were incubated with Cell Count Reagent SF (Nacalai Tesque Inc.) for 1-4 h. The absorbance of the resulting product at $450 \mathrm{~nm}$ was measured, and the background absorbance at $650 \mathrm{~nm}$ was subtracted. Experiments were conducted in triplicate.

Western blotting. Cells $\left(5 \times 10^{4}\right.$ cells $\left./ \mathrm{ml}\right)$ were cultured in 6-well plates (Corning, cat. no. 353046, $4 \mathrm{ml} /$ well) and treated with cSBL or control. Whole cell lysates were prepared using extraction buffer [150 mM NaCl, $10 \mathrm{mM}$ Tris-HCl (pH 7.4), 5 mM EDTA, 1\% Nonidet P-40, 0.1\% sodium deoxycholate, and $0.1 \%$ sodium dodecyl sulfate] supplemented with complete $^{\mathrm{TM}}$ Mini EDTA-free protease inhibitor cocktail tablets (one tablet/10 ml; Roche Applied Science). Soluble proteins were collected, and the protein concentration was measured using a BCA protein assay kit (Thermo Fisher Scientific, Inc., cat. no. 23227) according to the manufacturer's instructions. Proteins were separated using 10 or $14 \%$ SDS-PAGE and transferred onto Immobilon-P transfer membranes (Thermo Fisher Scientific, Inc.). The membranes were sequentially incubated with primary and secondary antibodies diluted in Can Get Signal solution (Toyobo Co., Ltd.). Protein bands were detected using ECL Prime Western Blotting Detection Reagent (Cytiva). The relative density of the protein bands was measured using ImageJ 1.51s software (National Institutes of Health). Experiments were repeated in triplicates.

Total RNA isolation. Total RNA was isolated from cells using TRI Reagent (Molecular Research Center) and purified using the SV Total RNA Isolation System (Promega) according to the manufacturer's instructions. RNA samples were quantified using an ND-1000 spectrophotometer (NanoDrop Technologies), and the quality was confirmed using a 2200 TapeStation (Agilent Technologies). The RNA integrity number equivalent $\left(\mathrm{RIN}^{\mathrm{e}}\right)$, which was an index of RNA degradation, was calculated from the $28 \mathrm{~S}$ and $18 \mathrm{~S}$ ribosomal RNA band peak values and other band peak values in the electrophoretic image. For the subsequent cDNA labeling, the Agilent Low-Input QuickAmp Labeling kit (Agilent Technologies, cat. no. 5190-2305) was used.

Gene expression microarrays. cDNA was amplified, labeled, and hybridized to a $60 \mathrm{~K}$ Agilent 60-mer oligomicroarray according to the manufacturer's instructions. All hybridized microarray slides were scanned using an Agilent scanner. Relative hybridization intensities and background hybridization values were calculated using Agilent Feature Extraction Software (9.5.1.1).

Data analysis and filter criteria. Raw signal intensities and flags for each probe were calculated from hybridization intensities (gProcessedSignal) and spot information (gIsSaturated, etc.), according to the procedures recommended by Agilent. [Flag criteria on GeneSpring Software was as follows: Absent (A): 'Feature is not positive and significant' and 'Feature is not above background;' Marginal (M):
'Feature is not Uniform,' 'Feature is Saturated,' and 'Feature is a population outlier;' and Present $(\mathrm{P})$ : others.]. The raw signal intensities of two samples were $\log 2$-transformed and normalized by quantile algorithm with the Bioconductor preprocessCore library package $(35,36)$. We selected probes that called the P flag in at least two samples. To identify up- and downregulated genes, we calculated Z-scores (37) and ratios (non-log scaled fold-change) from the normalized signal intensities of each probe to compare control and experimental samples. Then, we established the following criteria for differentially regulated genes: Upregulated genes: $Z$-score $\geq 2.0$ and ratio $\geq 1.5$-fold and downregulated genes: $Z$-score $\leq-2.0$ and ratio $\leq 0.66$. Data have been deposited in NCBI's Gene Expression Omnibus repository (38) (http://www.ncbi.nih.gov/geo) under the accession number: GSE 162286.

Functional annotation of DEGs in cSR cell lines. DEGs in cSR cells were characterized functionally using a hypergeometric test to find overrepresented gene ontology terms in the three main broad ontologies (biological process, molecular function, and cellular component) $(39,40)$. DEGs were also mapped to the Kyoto Encyclopedia of Genes and Genomes (KEGG) (41), which assigns proteins to pathways, to find overrepresented pathways. The analyses were done using the Database for Annotation, Visualization, and Integrated Discovery online tool (42).

Network analysis. GeneMANIA (43), an online database that identifies other proteins associated with a set of input genes, was used to generate protein-protein interaction (PPI) network images. The associations between co-expression, colocalization, predicted related genes, shared protein domains, genetic interactions, and physical interactions were determined using GeneMANIA.

Reverse transcription quantitative polymerase chain reaction $(R T-q P C R)$. mRNA expression of the top five downregulated genes (THY1, AKR1B15, AKR1B10, SLC47A2, and $C B R 1)$ were examined using RT-qPCR. Cells $\left(2 \times 10^{5}\right)$ were cultured for $48 \mathrm{~h}$, and total RNA was extracted using an AllPrep RNA/Protein kit (Qiagen, cat. no. 80404). cDNA was synthesized from total RNA $(1 \mu \mathrm{g})$ using a SuperScript VILO cDNA Synthesis Kit (Invitrogen, cat. no. 11754050). RT-qPCR was performed using a LightCycler 480 system with the LightCycler 480 Probes Master Kit (Roche Diagnostics, cat. no. 04707494001). PCR primers using a TaqMan/probe library assay were designed by the Universal Probe Library Assay Design Center (https://www.roche-applied-science. $\mathrm{com} / \mathrm{sis} / \mathrm{rtpcr} / \mathrm{upl} / \mathrm{acenter} . \mathrm{jsp})$. The expression levels of these genes were standardized relative to the mRNA expression level of GAPDH (as a housekeeping gene) based on their average cycle threshold values.

Statistical analysis. The results from three or more independent experiments were expressed as the mean \pm standard deviation. Statistical analyses were conducted using GraphPad Prism 5.0 (GraphPad Software, Inc.), and comparisons were made using one-way analysis of variance followed by Bonferroni's post hoc test. $\mathrm{P}<0.05$ was considered statistically significant. 


\section{Results}

Establishment of cSR cells. cSR cells were established by adding different cSBL concentrations at the low range to the culture medium. Five clones were obtained by limiting-dilution cloning. The sensitivity of the clones to cSBL was assessed using colony formation assay. As shown in Fig. 1A (upper panel), no colonies were found in parental $\mathrm{H} 28$ cells treated with cSBL at concentrations of $25 \mathrm{nM}$ or higher. In contrast, all five clones showed resistance to $\mathrm{cSBL}$. We calculated $\mathrm{IC}_{50}$ values from dose-response curves (Fig. 1B). The resistant rate (RR) represented the ratio of the $\mathrm{IC}_{50}$ value in cSR cells to the $\mathrm{IC}_{50}$ value in H28 cells (Fig. 1A, lower panel). cSR-A1 and cSR-B1 had the highest RRs (15.3 and 14.5, respectively); therefore, these two clones were utilized in subsequent experiments.

Analysis of $c S R$ cell lines characteristics. The growth curves of H28, cSR-A1, and cSR-B1 cells were examined (Fig. 2A). There was no significant difference in the growth rates among these three cell lines. However, cSR cells tended to show shrunken morphology in low-density culture conditions, even though no difference was observed in high-density culture conditions (Fig. 2B). The cSBL resistance was then evaluated. After treating cells with cSBL, ONC, or DOX for $72 \mathrm{~h}$, the cell viability was measured using the WST-8 assay, dose-response curves were prepared, and the $\mathrm{IC}_{50}$ values and RRs were calculated (Fig. 2C). The RRs of cSR cells to cSBL in the WST-8 assay were 4.4 (A1) and 4.6 (B1), which were lower than those obtained in the colony formation assay. Further, the RRs to ONC and DOX were lower than to cSBL; the RRs to ONC for cSR-A1 and cSR-B1 were 1.6 and 2.0, respectively, and the corresponding RRs to DOX were 0.7 and 1.0, respectively. Similar to what we observed for cSBL, cSR cells tended to show some resistance to RNA-targeted ONC. However, for DOX, a DNA-damaging anticancer drug, the RRs of cSR cells did not exceed 1, i.e., the effect of DOX was not different between parent $\mathrm{H} 28$ and cSR cells. Since it has been shown that the antitumor effect of cSBL was due to the induction of apoptosis, we examined whether the cSR cells had reduced apoptosis after cSBL treatment. Cells were treated with cSBL $(1$ or $5 \mu \mathrm{M})$ for $72 \mathrm{~h}$, and protein was extracted to examine the levels of cleaved caspase-3 (Fig. 2D). There was less cleaved caspase-3 in cSBL-treated cSR-B1 cells than in $\mathrm{H} 28$ cells, but the difference was not significant. Taken together, our results indicate that cSR cells become resistant to cSBL after long-term treatment with low concentrations, but they have relatively weak resistance to short-term treatment with high concentrations. In addition, there was no significant difference between $\mathrm{H} 28$ and cSR cells in terms of proliferation, morphology, or apoptosis after treatment with high cSBL concentrations.

Altered gene expression in cSR cell lines. Total RNA was extracted from H28, cSR-A1, and cSR-B1 cells and RNA quality was evaluated. All samples had RIN ${ }^{\mathrm{e}}$ values of 10.0 (Fig. S1), indicating that RNA could be extracted in a condition when it was hardly degraded. Therefore, these samples were used for microarray analyses. A comparison of the expression profiles in the cSR-A1, cSR-B1, and parental H28 cell lines revealed that 1254 genes (623 upregulated and 631 downregulated) were dysregulated in cSR-A1 cells and
A

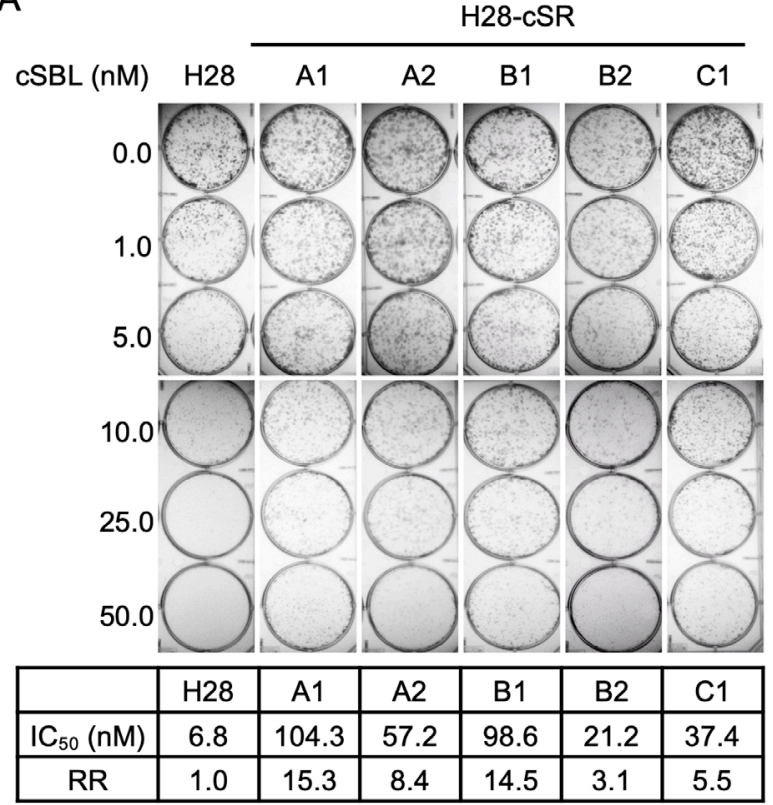

B

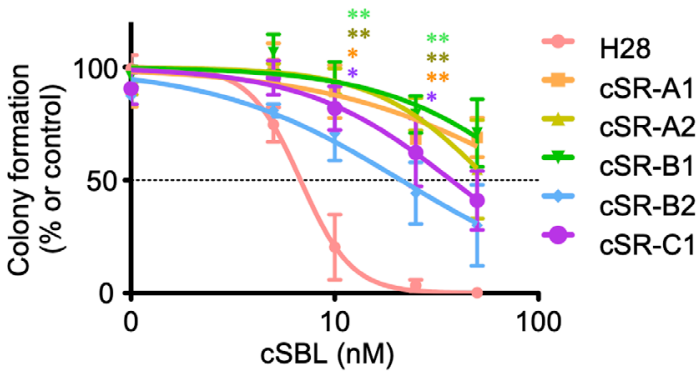

Figure 1. Effect of cSBL on colony formation in H28 and cSR cells (A) Colony formation assays are performed in the absence or presence of cSBL (1 to $50 \mathrm{nM}$ ). Representative images from three independent experiments are shown. $\mathrm{IC}_{50}$ values and $\mathrm{RRs}$ of each cell line are indicated below the images. (B) Colony numbers in (A) are counted and dose-response curves are depicted. Each data point represents the mean \pm SD of three independent experiment. The statistical significance of the colony formation of the cells compared to the control $\mathrm{H} 28$ cells were shown. ${ }^{*} \mathrm{P}<0.05,{ }^{* *} \mathrm{P}<0.01$ vs. H 28 . cSBL, bullfrog sialic acid-binding lectin; cSR, cSBL-resistant; RR, resistance rate; $\mathrm{SD}$, standard deviation.

1,225 genes (608 upregulated and 617 downregulated) were dysregulated in cSR-B1 cells compared to H28 cells. Among them, 927 genes (440 upregulated and 487 downregulated) were common DEGs out of 37,756 known coding transcripts on the microarray $(2.46 \%)$. The fold change ranged from 1.5- to 934.8-fold for upregulated genes and 1.5- to 755.7-fold for downregulated genes. The top 20 up- and downregulated genes in cSR cell lines are listed in Table SI.

GO enrichment analysis. To further understand the functional relevance of DEGs in cSR cell lines, we performed gene ontology analysis. The 927 DEGs were used to extract the associated ontologies based on three broad ontology categories: 'biological process,' 'molecular function,' and 'cellular component.' In all cases, a P-value $\leq 0.05$ was considered statistically significant. The 20 most enriched GO terms for the DEGs are listed in Table SII. There were 123 significantly enriched GO terms in the biological process category. 
A

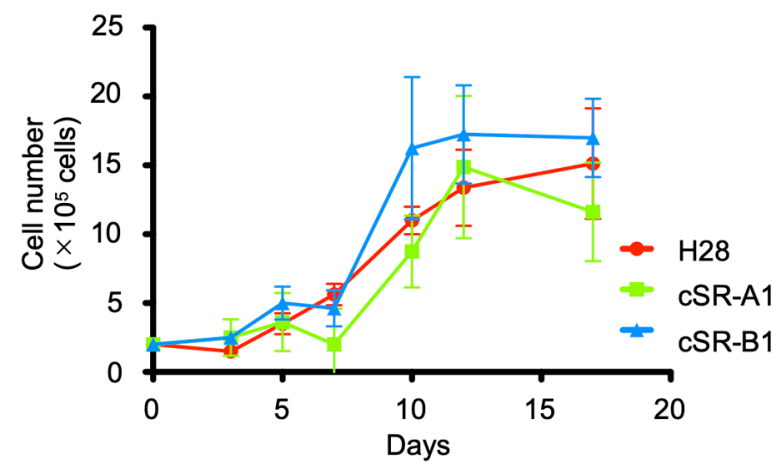

C
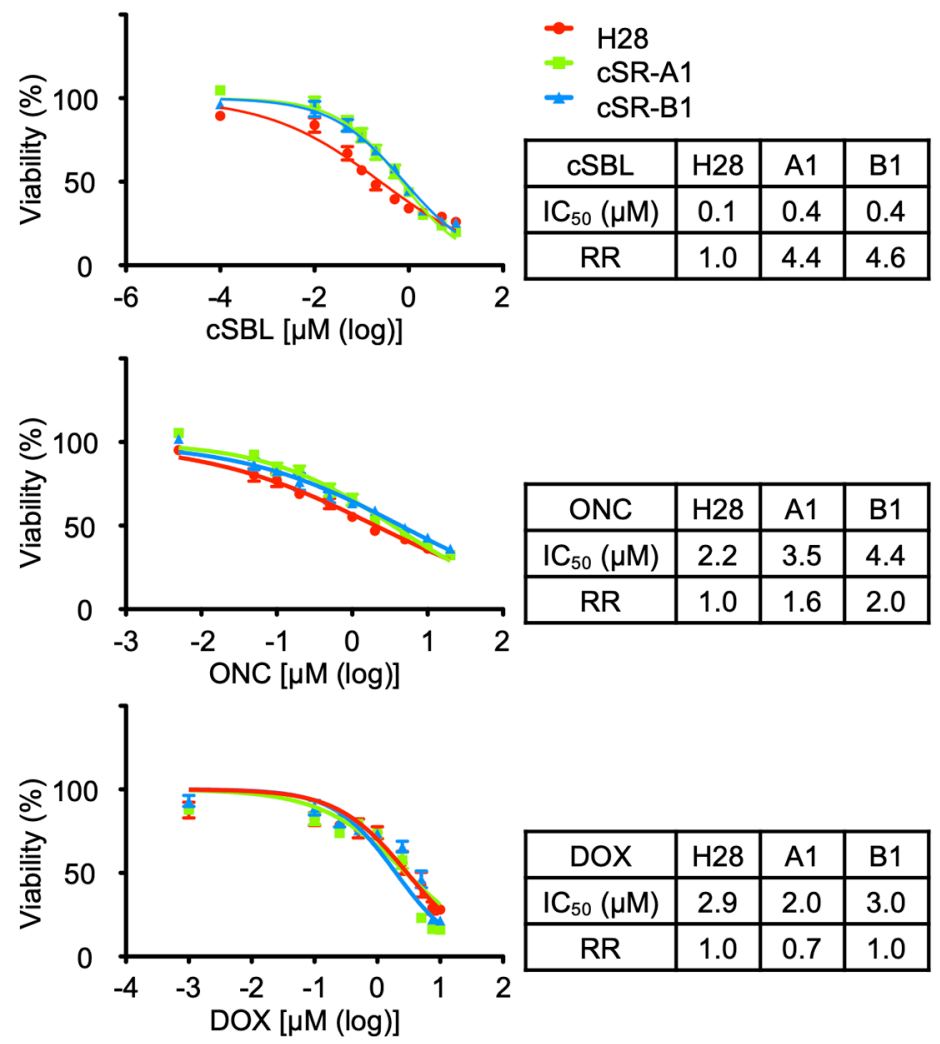

B
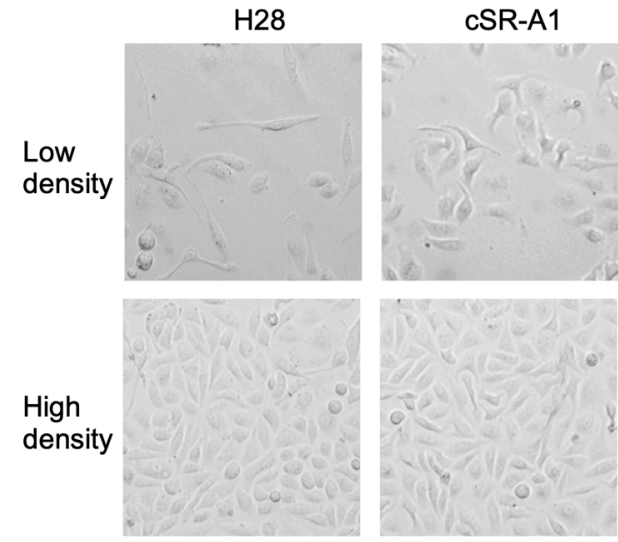

CSR-B1

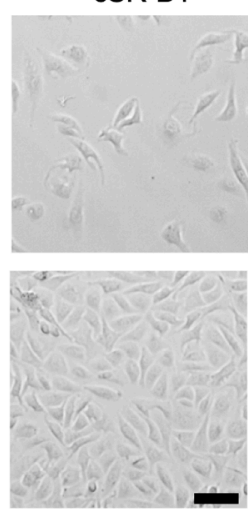

Magnification ; $10 \times($ scale : $100 \mu \mathrm{m})$

D
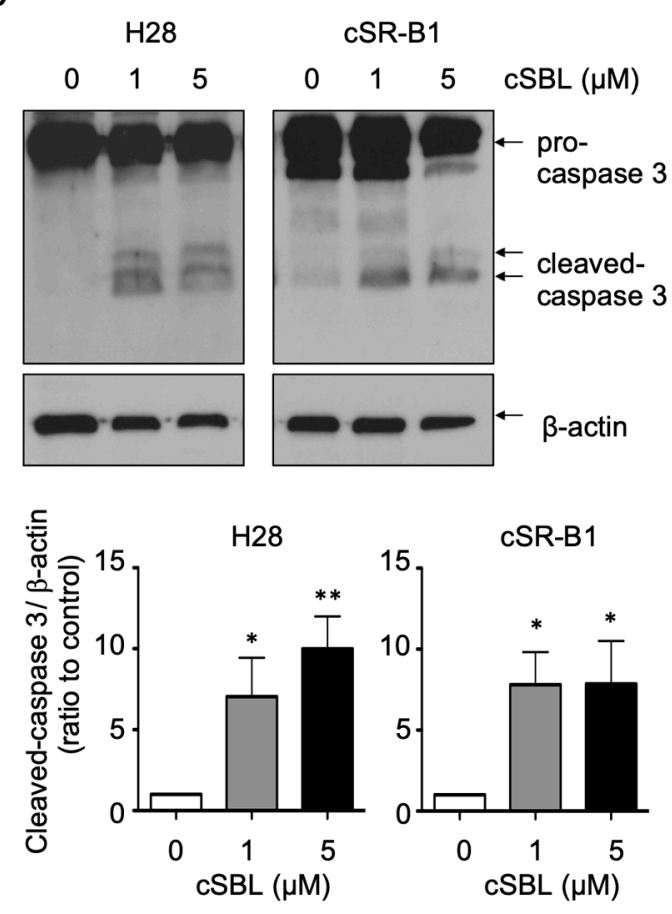

Figure 2. Analysis of cSR cell line characteristics. (A) Growth of $\mathrm{H} 28$ and cSR cells. Cells are seeded at $2 \times 10^{5}$ cells/well and counted at the timepoints (3 to 17 days) as indicated. Each data point represents the mean \pm SD of three independent experiment. (B) Morphology of H28 and cSR cells. Cells are seeded at $1 \times 10^{5}$ cells/well. After $24 \mathrm{~h}$ (low density) or $72 \mathrm{~h}$ (high density), cell morphology is observed. Scale bar, $100 \mu \mathrm{m}$. (C) Effect of cSBL, ONC, and DOX on $\mathrm{H} 28$ and cSR cells. Viability of cells treated with the drugs are measured by the WST- 8 assay. $\mathrm{IC}_{50}$ values and RRs are calculated from the depicted dose-response curves. Each data point represents the mean $\pm \mathrm{SD}$ of three independent experiment performed in triplicates. (D) Apoptosis induction in H28 and cSR-B1 cells treated with cSBL. Cells are treated with cSBL $(1$ or $5 \mu \mathrm{M})$, and the expression of cleaved caspase-3 is detected using western blotting. Densitometric quantification is performed using the results of three independent experiments (mean $\pm \mathrm{SD}$ ). The statistical significance of the bands compared to the non-treated control were shown. ${ }^{*} \mathrm{P}<0.05,{ }^{* *} \mathrm{P}<0.01$ vs. $0 \mu \mathrm{M}$ cSBL. cSR, cSBL-resistant; cSBL, bullfrog sialic acid-binding lectin; ONC, onconase; DOX, doxorubicin; RR, resistance rate; $\mathrm{SD}$, standard deviation.

The most significant term was oxidation-reduction process (GO:0055114). Interestingly, the GO terms included not only terms related to cancer characteristics, such as cell proliferation (GO:0008284, GO:0008285), adhesion (GO:0007155, GO:0007162), migration (GO:0016477), and apoptosis (GO:0006915), but also several metabolic processes related to lipids (GO:0006869), cellular protein (GO:0044267), and drugs (GO:00171449). In the molecular function category, 36 functions were enriched, and the most significantly enriched term was integrin binding (6.87E-07). There were several other binding functions, including growth factors [IGF (GO:0005520), FGF (GO:0017134), TGF (GO:0050431), and EGF (GO:0005154)] and other cell membrane molecules such as receptors (GO:0005102), heparin (GO:0005102), and syndecan (GO:0045545). Thirty-two components were enriched in the cellular component category. The top four GO terms in cellular component included 'extracellular' [extracellular space (GO:0005615), extracellular exosome 


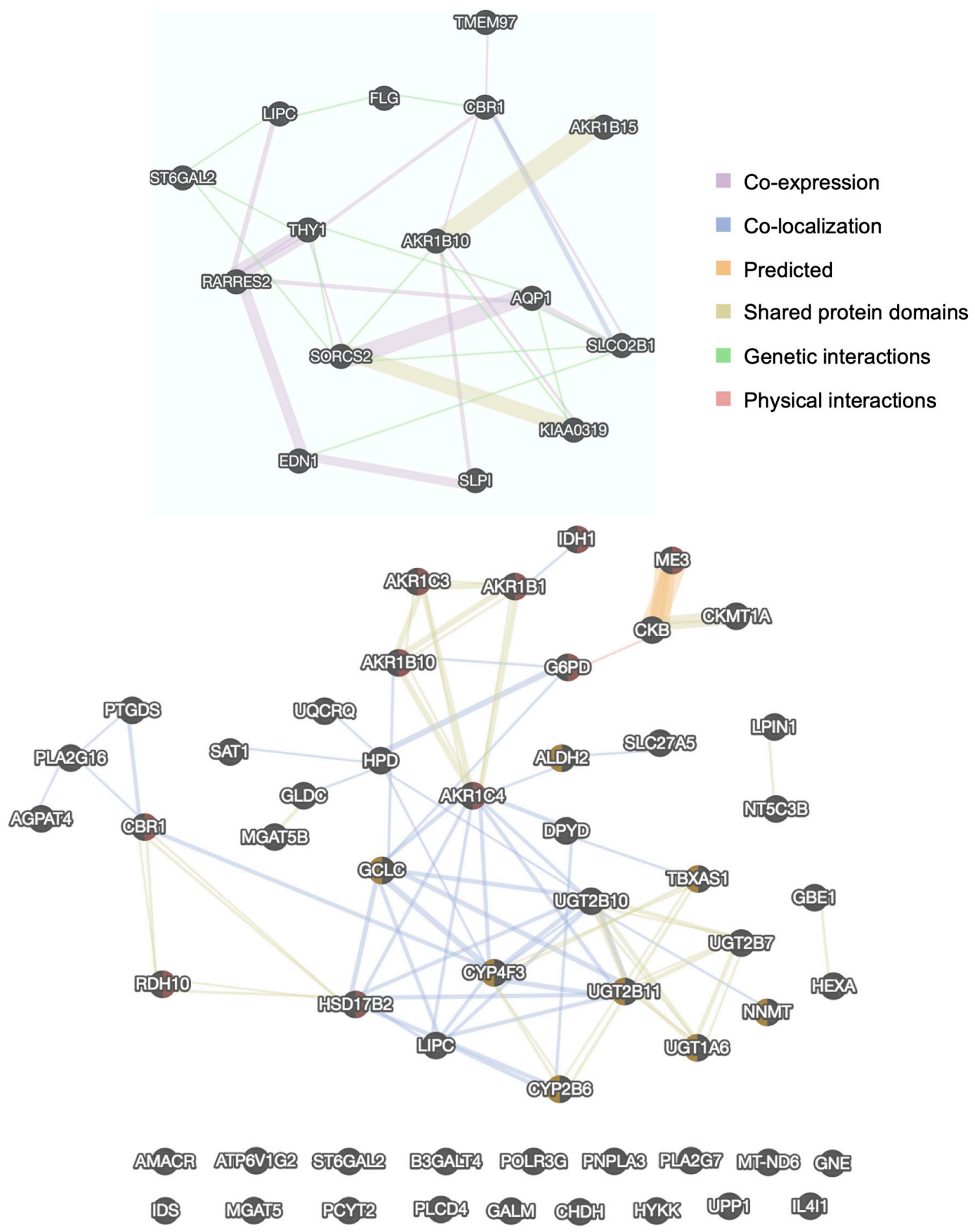

Figure 3. The protein-protein interaction networks of the top 10 up- and downregulated DEGs (upper panel) and the 57 genes involved in metabolic pathways (lower panel). Different colors represent diverse bioinformatics methods. DEGs, differentially expressed genes.

(GO:0070062), extracellular matrix (GO:0031012), and extracellular region (GO:0005576)]. They were followed by terms related to the cell membrane, such as cell surface (GO:0009986), basement membrane (GO:0005604), and plasma membrane (GO:0005886). Altogether, it appears that there are many differences between parental $\mathrm{H} 28$ cells and cSR cells in cellular functions, especially in association with cancer characteristics and metabolic processes. Extracellularand cell membrane-associated GO terms were highly enriched in the DEGs of cSR cell lines.
Pathway analysis. To analyze the signaling pathways affected by DEGs in cSR cell lines, we analyzed DEGs using the KEGG database. Table SIII shows the 18 significantly affected pathways in cSR cell lines. Among the significantly enriched pathways (determined by a hypergeometric test where $\mathrm{P}<0.05$ ), "metabolism of xenobiotics by cytochrome P450' was the most significant. Additionally, there were several pathways involved in various metabolic processes. Fifty-seven genes were involved in metabolic pathways, all related to lipid and carbohydrate metabolism. These results 

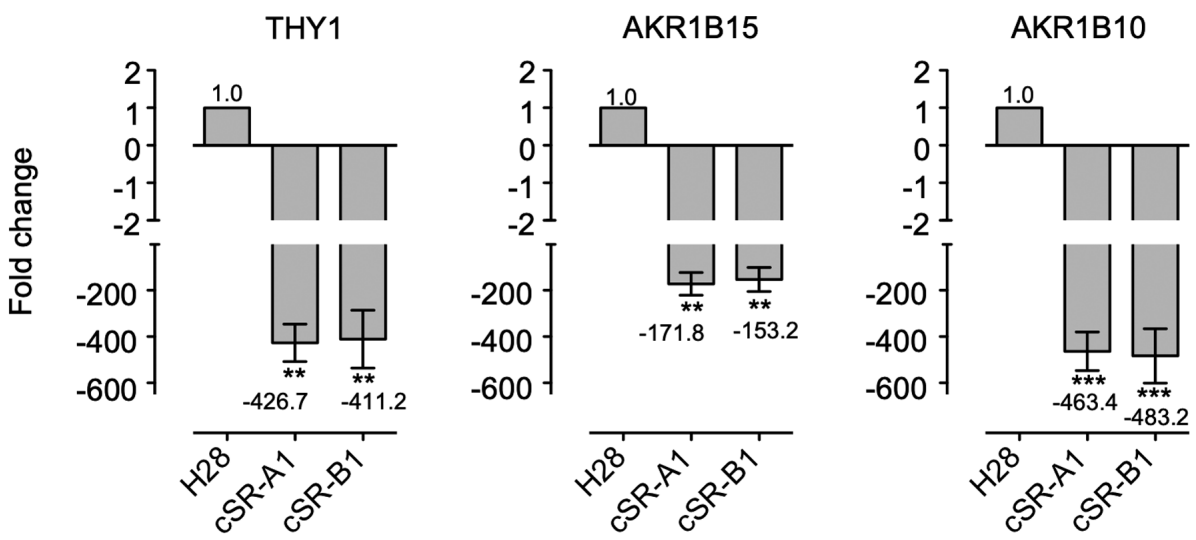

SLC47A2

CBR1

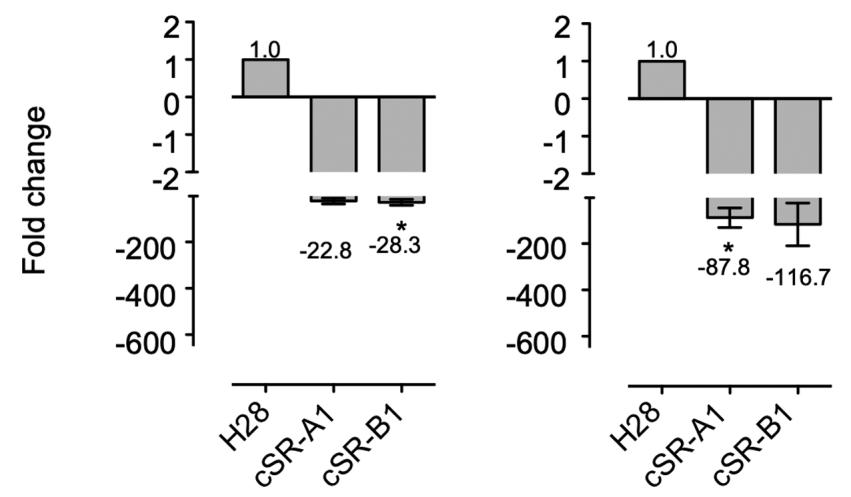

Figure 4. Expression of THY1, AKR1B15, AKR1B10, SLC47A2, and CBR1 mRNA in H28 and cSR cells. Quantitative RT-PCR is performed using specific primers and GAPDH (control gene). The expression levels of genes are normalized to the level of GAPDH, and the level of the corresponding gene in $\mathrm{H} 28$ cells (control cell line) is set at 1 . Data are presented as mean $\pm \mathrm{SD}$ of three independent experiment performed in triplicates, and the mean values are indicated on the $\mathrm{X}$ axis titles. ${ }^{*} \mathrm{P}<0.05,{ }^{* *} \mathrm{P}<0.01,{ }^{* * * *} \mathrm{P}<0.001$ vs. $\mathrm{H} 28$. cSR, bullfrog sialic acid-binding lectin-resistant; RT-PCR, reverse transcription polymerase chain reaction; SD, standard deviation; THY1, Thy-1 Cell Surface Antigen (CD90); AKR, aldo-keto reductase; SLC47A2, solute carrier family 47 member 2; CBR1, carbonyl reductase; GAPDH, glyceraldehyde-3-phosphate dehydrogenase.

prompted our interest in further analysis of the interactions among these DEGs.

PPI network analysis. The networks of proteins encoded by the top 10 up- and downregulated DEGs and the 57 genes in the KEGG metabolic pathways were identified using the GeneMANIA PPI network (Fig. 3). The color of the line represents the type of interaction, and the size of the node indicates the degree of interaction in the PPI, where larger nodes have more interactions. These data demonstrated there were some protein families whose expression was affected in cSR cells, such as AKR or UDP-glucuronosyltransferase. However, there were no significant hub proteins in either PPI network, suggesting that long-term exposure of cSBL affected gene expression in a pleiotropic fashion.

$R T$ - $q P C R$ analysis. As indicated above, we found that the expressions of some AKR family members were affected in cSR cells (Fig. 3). Two of these genes, $A K R 1 B 15$ and $A K R 1 B 10$, were among the top three most downregulated genes in cSR cells (Table SI). Therefore, we were interested in the AKR family, which has been reported to be associated with cancer, and conducted subsequent studies focusing on highly downregulated genes in cSR cells. In order to confirm the reproducibility of the microarray, we compared gene expression patterns between parental and cSR cells using RT-qPCR. As shown in Fig. 4, THY1, AKR1B15, AKR1B10, $S C L 47 A 2$, and $C B R 1$ all had reduced expression in CSR-A1 and cSR-B1 cells. These genes had 22.8- to 483.2-fold decreased expression, and this downregulation was similar in both cSR-A1 and cSR-B1 cells. Although these fold changes were different from those observed in the microarray analysis, they were in the same direction. Therefore, RT-qPCR confirmed that the microarray experiments were valid and showed that the changes were highly significant.

Confirmation of AKR1B10 downregulation using western blot. Next, we further examined protein expression of the AKR family members that were downregulated in cSR cells. We found that the expression of six AKR family genes were decreased from 9.1- to 562.0-fold in the microarray analysis (Table I). We focused on AKR1B10, which has been reported to be associated with cancer (44) and whose antibody was commercially available. The expression of AKR1B10 at the protein level was confirmed using western blotting. A significant decrease in AKR1B10 expression was observed in cSR-A1 and cSR-B1 cells compared to H28 cells (Fig. 5). Therefore, the decreased expression of AKR1B10 as detected by the microarray analysis was also observed at the protein level. 
Table I. Gene expression changes of AKR family in cSR cells.

\begin{tabular}{lc}
\hline Gene symbol & Fold change $^{\mathrm{a}}$ \\
\hline AKR1B15 & -562.0 \\
AKRlB10 & -548.5 \\
AKRlC1 & -54.0 \\
AKRlC4 & -24.7 \\
AKRlB1 & -11.4 \\
AKRIC3 & -9.1 \\
\hline
\end{tabular}

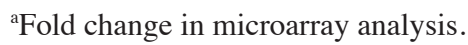

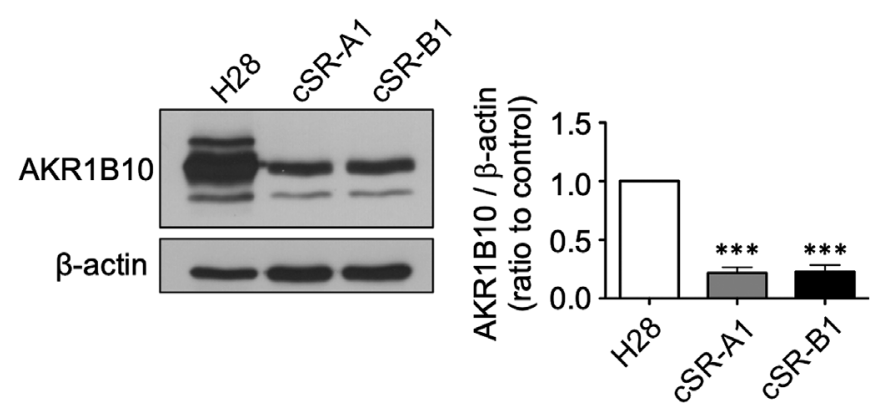

Figure 5. AKR1B10 protein expression in H28 and cSR cells. The expression of AKR1B10 in each cell line is detected using western blotting. Densitometric quantification is performed using the results of three independent experiments (mean $\pm \mathrm{SD}$ ). ${ }^{* * *} \mathrm{P}<0.001$ vs. H28. cSR, bullfrog sialic acid-binding lectin-resistant; SD, standard deviation.

Further investigation of DEGs other than the AKR family in $c S R$ cells. Finally, we further investigated genes that showed expression fluctuations based on the results of microarray analysis. We found that the expression of some members of ATP-binding cassette (ABC) transporter superfamily was decreased in cSR cells (Table SIV). The expression of $A B C C 2$ was -17.3 times lower and $A B C A 1$ was -4.7 times lower in cSR cells.

\section{Discussion}

In this study, we produced cSR cells by treating sensitive $\mathrm{H} 28$ cells with low doses of cSBL over a long period. We also succeeded in identifying genes whose expression was altered in cSR cells (Table SI). The GO analysis and pathway analysis indicated that these genes were related to cell proliferation and cell membrane, as well as sugar and lipid metabolism (Tables SII and SIII). We also found that AKR1B10 was significantly reduced in cSR cells at the protein level (Fig. 5). These findings indicate that long-term treatment of cancer cells with cSBL alters many metabolic genes and reduces the expression of AKR family genes, revealing a new aspect of the antitumor effect of cSBL.

Analysis of cSR cell lines characteristics revealed that although cSR cells tended to exhibit shrunken morphology in low-density culture conditions, we could not detect significant differences in morphology in high-density culture conditions, proliferation or apoptosis after treatment with high cSBL concentrations (Fig. 2). The reason for the morphological change observed in low-density culture conditions is unclear, but we speculate that this change may have been caused by changes in expression of genes related to the morphology, adhesion or migration. Indeed, GO enrichment analysis showed there were some enriched GO terms such as cell surface and plasma membrane in cellular component category or integrin binding in molecular function category. The RRs to cSR were high (15.3 and 14.5 for cSR-A1 and -B1, respectively) in the colony assay but low (4.4 and 4.6 for cSR-A1 and -B1, respectively) in the WST-8 assay (Figs. 1 and 2). By the nature of the experiment, the colony assay observed colony forming ability for a relatively long time (12 days) in the presence of low concentrations of cSBL (1-50 $\mathrm{nM})$ and the WST-8 assay monitored survival changes in the presence of high concentrations of $\operatorname{cSBL}(0.01-10 \mu \mathrm{M})$ for a short period of time $(72 \mathrm{~h})$. This indicates that cSR cells are resistant to long-term treatment with low concentrations of cSBL, but under short-term treatment conditions with relatively high concentrations, the apoptosis-inducing effect of cSBL is also observed in cSR cells. In other words, it is suggested that even in cSR cells that have acquired resistance by some mechanism in this long-term treatment, intracellular RNA is cleaved and apoptosis is induced by cSBL treatment exceeding a certain concentration. The dramatic mechanism of action that involves RNA cleavage may interfere with the ability of cancer cells to develop high resistance to cSBL.

Experiments that require RNA extracted from cytotoxic RNase-treated cells, such as RNA expression analysis, are difficult to perform. Such experiments often have low accuracy because the extracted RNA is presumably not intact in the cells that are treated with cytotoxic doses of RNase, and the RNase may degrade RNA even during the cell lysing step. However, several recent reports identified DEGs in cytotoxic RNase-treated cells using microarray technology. These experiments were carried out in very strict conditions, such as including only RNA with acceptable concentrations and $\mathrm{A}_{260 / 280}$ ratios or high RIN values, as it was done in this experiment (32-34). Some of the identified genes whose expression was affected by cSBL treatment were also identified in a microarray examining cells treated with PE5. Since we used resistant cells, the identified DEGs may have include genes involved in the resistance to cSBL. However, the decrease in the AKR family detected here was also observed in the short-term treatment of PE5; PE5 is known to reduce the expression of AKR1A1, a member of the AKR family (34). Therefore, the downregulation of AKR family members might be a universal response of cancer cells to cytotoxic RNase or involved in the antitumor effects of cytotoxic RNases. The microarray analyses revealed that there were significant pleiotropic changes including those in the expression of multiple genes involved in metabolic pathways in cSBL-resistant cells (Table SIII). Some of these metabolic pathway related genes are listed among the top 20 list of genes up- or down-regulated in cSR cells (Table SI). Among the up-regulated genes, the increase in expression of LIPC which catalyzes the hydrolysis of triglycerides and phospholipids (45), was the highest (934.8 fold higher in cSR cells, Table SI). ST6GAL2, which showed the third largest change (126.7 fold higher in cSR cells, Table SI), is an enzyme that transfers sialic acid from the donor of substrate CMP-sialic acid to galactose containing acceptor substrates (46). It is interesting to note that the expression 
level of this enzyme was elevated, because the presence of sialic acids at the cell surface is thought to be important for the effect of cSBL (20). In this study, because we found that the expressions of some AKR family members were affected in cSR cells, further investigations were focused mainly on strongly downregulated genes in cSR cells.

The AKR superfamily is a family of enzymes that reversibly reduce carbonyl groups (47). These proteins catalyze a variety of metabolic oxidation-reduction reactions, including reduction of glucose, glucocorticoids, small carbonyl metabolites, glutathione conjugates, and phospholipid aldehydes (48). More than 150 proteins belonging to this superfamily are classified into 15 families (AKR1 to AKR15) based on the similarity of amino acid sequences. Each family is further subdivided into subfamilies, which have $60 \%$ or higher similarity at the amino acid level (47). The largest family, AKR1, is subdivided into six subfamilies (AKR1A, AKR1B, AKR1C, AKR1D, AKR1E, and AKR1G). In humans, there are 14 AKR superfamily proteins, nine of which belong to the AKR1 family (49). Our microarray analysis revealed that multiple AKR genes were downregulated in cSR cells. In addition, when we focused on AKR1B10, which is involved in resistance to anticancer drugs and has attracted attention as a new target in cancer therapy (49), we found that its expression was significantly reduced at the protein level in cSR cells. AKR1B10 has been reported to be overexpressed in lung cancer (50), liver cancer $(51,52)$, breast cancer (50), pancreatic cancer (53), and oral squamous cell carcinoma (54). One of the roles of AKR1B10 in cancer cells is to suppress the production of retinoic acid, a cell differentiation-promoting factor. Retinoic acid is produced from retinol via retinal and binds to the nuclear receptors, retinoic acid receptor and retinoid $\mathrm{X}$ receptor, to promote cell differentiation (55). AKR1B10 is thought to promote cancer cell proliferation and survival by reducing retinal to retinol, thereby decreasing intracellular retinoic acid production $(56,57)$. In addition, AKR1B10 promotes cancer cell survival by reducing cytotoxic aldehydes produced by lipid peroxidation, such as 4-hydroxynonenal $(58,59)$, and is involved in resistance to anticancer drugs such as cisplatin, mitomycin $\mathrm{C}$, anthracyclines (doxorubicin and idarubicin), and docetaxel (60-62). Therefore, AKR1B10 has potential not only as a cancer biomarker but also as a novel therapeutic target for cancer treatment and may promote chemosensitization. Although reports on AKR1B10 in MPM are very limited, AKR1B10 may also be associated with malignancy in MPM, as in the other cancer cases indicated above. A study aimed to search for novel biomarkers in malignant mesothelioma performed by Mundt et al (63), identified that AKR1B10 was one of the prognostic mesothelioma biomarker candidates. Patients with high AKR1B10 levels had a mean survival time that was 5.5 months shorter than that in patients with low AKR1B10 expression levels (5.5 vs. 11.0 months, respectively; $\mathrm{N}=14$ for high and 13 for low expression level). Usami et al (64) established two morphologically distinct MPM cell lines, Y-MESO-8A (epithelial-like) and Y-MESO-8D (spindle-like) from the same patient. Microarray analysis to determine differences in gene expression in these cells showed that the expression of $A K R 1 B 10$ and $A K R 1 C 3$ in Y-MESO-8D were 17.8 and 6.35 times higher, respectively, than that of Y-MESO-8A (64). Another report showed that under serum starvation conditions, AKT was phosphorylated in Y-MESO-8D but not in Y-MESO-8A (65). However, there are no reports related to the function of AKR1B10 in those cells. Detailed investigations focusing on the function of AKR1B10 in MPM are needed. It is interesting to note that decreased AKR1B10 expression was observed at the protein level in cells established by long-term exposure to cSBL. It is possible that cSBL could be used to promote chemosensitivity to anticancer drugs. Indeed, Toyooka and Hayakawa's group has succeeded in developing a novel AKR1B10 inhibitor that suppressed cisplatin resistance in non-small cell lung cancer cells. It also blocked the proliferative and metastatic potential in these cells (66). Now we are working on the comprehensive investigation of the effect of cSBL on AKR family including impact of several anticancer drugs in cSR cells,

In addition to $A K R 1 B 10$, the expression levels of $A K R 1 B 1$, $A K R 1 C 1, A K R 1 C 3$ and $A K R 1 C 4$ were reduced in $\mathrm{cSR}$, and it has also been reported that they were involved in resistance to cisplatin, daunorubicin or DOX $(52,67,68)$. Furthermore, we found that the expression of some members of the ATP-binding cassette $(\mathrm{ABC})$ transporter superfamily, which contributed to chemotherapeutic resistance, was reduced in cSR cells (Table SIV). In humans, there are 49 known ABC genes classified into seven different families (A-G) depending on their amino acid sequence (68). They serve a variety of functions other than drug resistance and can be expressed as channels, receptors and transporters (69). The members involved in drug efflux from human cells do not belong to one particular family (69). Among the ABC transporters found to be reduced in cSR cells, ABCC2 has been reported to contribute to resistance against methotrexate, doxorubicin, cisplatin (68), and ABCA1 is responsible for transporting cholesterol and phospholipids (70), but has also been reported to contribute to resistance to Nitidine, a cytotoxic benzophenanthridine alkaloid (71). Shukla et al reported that several ABC transporter genes were endogenously overexpressed in three MPM cell lines as compared to untransformed LP9/TERT1 mesothelial cells (ABCB1 in MO, ABCC3 in ME-26, and ABCA2, ABCC5 and ABCA7 in HMESO cells) (9). Hudson et al (72) compared expression of genes involved in the response to chemotherapy between II-45 rat MPM cells and normal 4/4 RM.4 mesothelial cells, and between established chemo-resistant cell lines and parental II-45 cells, respectively. They found that ABCB1 and ABCG2 were endogenously overexpressed in II-45 MPM cells compared to 4/4 RM.4 normal cells; furthermore, levels of ABCB1 in cisplatin resistant II-45 cells, and ABCC2 in pemetrexed or combination (cisplatin plus pemetrexed) resistant II-45 cells were significantly increased compared to parental II-45 cells. Those reports indicate that although the molecular species which are involved in the chemoresistance differ depending on the cell type, ABC transporter superfamily members are associated with inherent and acquired drug resistance in MPM. Furthermore, ABCB5 is now considered as one of a therapeutic target in MPM, because ABCB5 is upregulated in MPM-initiating cells generated from primary MPM samples (73). Our previous studies have shown that cSBL had a stronger apoptosis-inducing effect on multidrug resistant K562 leukemia cells that overexpressed ABCB1 than on their parent K562 cells (27). Since we found that the expression of $A B C C 2$ was reduced in cSR, it was suggested that cSBL was effective 
against MPM regardless of intrinsic drug resistance and may be able to reduce MPM resistance to chemotherapeutic agents that are substrates for ABCC2. Indeed, although the difference was not statistically significant in our experimental conditions, cSR-A1 tended to be more sensitive to DOX (Fig. 2).

In conclusion, we found that long-term treatment with cSBL affected malignant mesothelioma cells by dysregulating multiple genes. The detected DEGs may include genes other than those directly affected by the cSBL application. Currently, examinations of the direct effect of cSBL treatment and combination research with other drugs are being conducted. Because cSBL significantly reduced the expression of AKR family members, especially AKR1B10, it may offer new possibilities for cancer therapy. We believe that investigation of other genes whose expression was changed in cSR cells will further elucidate the antitumor effect of cSBL. Furthermore, by enhancing the effect of cSBL itself and searching for effective concomitant drugs using information obtained in this study, our results can be expected to lead to the establishment of novel, more effective cancer treatments.

\section{Acknowledgements}

Not applicable.

\section{Funding}

This research was funded by a Grant-in-Aid for Young Scientists (B) (grant no. 17K15029) to Takeo Tatsuta.

\section{Availability of data and materials}

The datasets used and/or analyzed in the current study are available from the corresponding author on reasonable request.

\section{Authors' contributions}

TT and MH conceived and designed the study. TT, AN and SS acquired and analyzed the data. TT and MH confirmed the authenticity of all the raw data. TT prepared the draft of the manuscript, including the figures. All authors read and approved the final manuscript.

\section{Ethics approval and consent to participate}

Not applicable.

\section{Patient consent for publication}

Not applicable.

\section{Competing interests}

The authors declare that they have no competing interests.

\section{References}

1. Cakiroglu E and Senturk S: Genomics and functional genomics of malignant pleural mesothelioma. Int J Mol Sci 21: 6342, 2020.
2. Cugell DW and Kamp DW: Asbestos and the pleura: A review. Chest 125: 1103-1117, 2004.

3. Thanh TD, Van Tho N, Lam NS, Dung NH, Tabata C and Nakano Y: Simian virus 40 may be associated with developing malignant pleural mesothelioma. Oncol Lett 11: 2051-2056, 2016.

4. Farioli A, Ottone M, Morganti AG, Compagnone G, Romani F, Cammelli S, Mattioli S and Violante FS: Radiation-induced mesothelioma among long-term solid cancer survivors: A longitudinal analysis of SEER database. Cancer Med 5: 950-959, 2016.

5. Yap TA, Aerts JG, Popat S and Fennell DA: Novel insights into mesothelioma biology and implications for therapy. Nat Rev Cancer 17: 475-488, 2017.

6. Van Meerbeeck JP, Gaafar R, Manegold C, Van Klaveren RJ, Van Marck EA, Vincent M, Legrand C, Bottomley A, Debruyne C, Giaccone G, et al: Randomized phase III study of cisplatin with or without raltitrexed in patients with malignant pleural mesothelioma: An intergroup study of the European organisation for research and treatment of cancer lung cancer group and the National Cancer Institute. J Clin Oncol 23: 6881-6889, 2005 .

7. Vogelzang NJ, Rusthoven JJ, Symanowski J, Denham C, Kaukel E, Ruffie P, Gatzemeier U, Boyer M, Emri S, Manegold C, et al: Phase III study of pemetrexed in combination with cisplatin versus cisplatin alone in patients with malignant pleural mesothelioma. J Clin Oncol 21: 2636-2644, 2003.

8. Wright K: FDA approves nivolumab plus ipilimumab for the treatment of advanced HCC. Oncology (Willist Park) 34: 693606, 2020.

9. Shukla A, Hillegass JM, MacPherson MB, Beuschel SL, Vacek PM, Pass HI, Carbone M, Testa JR and Mossman BT: Blocking of ERK1 and ERK2 sensitizes human mesothelioma cells to doxorubicin. Mol Cancer 9: 314, 2010.

10. Pouliquen DL, Nawrocki-Raby B, Nader J, Blandin S, Robard M, Birembaut $\mathrm{P}$ and Grégoire $\mathrm{M}$ : Evaluation of intracavitary administration of curcumin for the treatment of sarcomatoid mesothelioma. Oncotarget 8: 57552-57573, 2017.

11. Makarov AA and Ilinskaya ON: Cytotoxic ribonucleases: Molecular weapons and their targets. FEBS Lett 540: 15-20, 2003.

12. Fang EF and Ng TB: Ribonucleases of different origins with a wide spectrum of medicinal applications. Biochim Biophys Acta 1815: 65-74, 2011.

13. Wus Y, Mikulskiq SM, Ardeltll W, Rybakt SM and Youlet RJ: A cytotoxic ribonuclease. J Biol Chem 268: 10686-10693, 1993.

14. Bosch M, Benito A, Ribó M, Puig T, Beaumelle B and Vilanova M: A nuclear localization sequence endows human pancreatic ribonuclease with cytotoxic activity. Biochemistry 43: 2167-2177, 2004

15. Balandin TG, Edelweiss E, Andronova NV, Treshalina EM, Sapozhnikov AM and Deyev SM: Antitumor activity and toxicity of anti-HER2 immunoRNase scFv 4D5-dibarnase in mice bearing human breast cancer xenografts. Invest New Drugs 29: 22-32, 2011.

16. Nitta K, Ozaki K, Tsukamoto Y, Hosono M, Ogawakonno Y, Kawauchi H, Takayanagi Y, Tsuiki S and Hakomori S: Catalytic lectin (leczyme) from bullfrog (Rana catesbeiana) eggs: Mechanism of tumoricidal activity. Int J Oncol 9: 19-23, 1996.

17. Nitta K, Takayanagi G, Kawauchi $\mathrm{H}$ and Hakomori S: Isolation and characterization of Rana catesbeiana lectin and demonstration of the lectin-binding glycoprotein of rodent and human tumor cell membranes. Cancer Res 47: 4877-83, 1987.

18. Nitta K, Ozaki K, Tsukamoto Y, Furusawa S, Ohkubo Y, Takimoto H, Murata R, Hosono M, Hikichi N, Sasaki K, et al: Characterization of a Rana catesbeiana lectin-resistant mutant of leukemia P388 cells. Cancer Res 54: 928-934, 1994.

19. Titani K, Takio K, Kuwada M, Nitta K, Sakakibara F, Kawauchi H, Takayanagi G and Hakomori S: Amino acid sequence of sialic acid binding lectin from frog (Rana catesbeiana) eggs. Biochemistry 26: 2189-2194, 1987.

20. Nitta K, Ozaki K, Ishikawa M, Furusawa S, Hosono M, Kawauchi H, Sasaki K, Takayanagi Y, Tsuiki S and Hakomori S: Inhibition of cell proliferation by Rana catesbeiana and Rana japonica lectins belonging to the ribonuclease superfamily. Cancer Res 54: 920-927, 1994.

21. Tatsuta T, Sugawara S, Takahashi K, Ogawa Y, Hosono M and Nitta K: Cancer-selective induction of apoptosis by leczyme. Front Oncol 4: 139, 2014.

22. Tatsuta T, Hosono M, Ogawa Y, Inage K, Sugawara S and Nitta K: Downregulation of Hsp70 inhibits apoptosis induced by sialic acid-binding lectin (leczyme). Oncol Rep 31: 13-18, 2014. 
23. Tatsuta T, Sugawara S, Takahashi K, Ogawa Y, Hosono M and Nitta K: Leczyme: A new candidate drug for cancer therapy. Biomed Res Int 2014: 421415, 2014.

24. Chen JN, Yiang GT, Lin YF, Chou PL, Wu TK, Chang WJ, Chen $\mathrm{C}$ and Yu YL: Rana catesbeiana ribonuclease induces cell apoptosis via the caspase-9/-3 signaling pathway in human glioblastoma DBTRG, GBM8901 and GBM8401 cell lines. Oncol Lett 9: 2471-2476, 2015.

25. Tatsuta T, Sato S, Sato T, Sugawara S, Suzuki T, Hara A and Hosono M: Sialic acid-binding lectin from bullfrog eggs exhibits an anti-tumor effect against breast cancer cells including triple-negative phenotype cells. Molecules 23: 2714, 2018

26. Tatsuta T, Satoh T, Sugawara S, Hara A and Hosono M: Sialic acid-binding lectin from bullfrog eggs inhibits human malignant mesothelioma cell growth in vitro and in vivo. PLoS One 13: e0190653, 2018.

27. Tatsuta T, Hosono M, Sugawara S, Kariya Y, Ogawa Y, Hakomori S and Nitta K: Sialic acid-binding lectin (leczyme) induces caspase-dependent apoptosis-mediated mitochondrial perturbation in Jurkat cells. Int J Oncol 43: 1402-1412, 2013.

28. Tatsuta T, Hosono M, Miura Y, Sugawara S, Kariya Y, Hakomori S and Nitta K: Involvement of ER stress in apoptosis induced by sialic acid-binding lectin (leczyme) from bullfrog eggs. Int J Oncol 43: 1799-1808, 2013

29. Kariya Y, Tatsuta T, Sugawara S, Kariya Y, Nitta K and Hosono M: RNase activity of sialic acid-binding lectin from bullfrog eggs drives antitumor effect via the activation of p38 MAPK to caspase3/7 signaling pathway in human breast cancer cells. Int J Oncol 49: 1334-1342, 2016.

30. Tatsuta T, Hosono M, Takahashi K, Omoto T, Kariya Y, Sugawara S, Hakomori S and Nitta K: Sialic acid-binding lectin (leczyme) induces apoptosis to malignant mesothelioma and exerts synergistic antitumor effects with TRAIL. Int J Oncol 44: 377-384, 2014

31. Satoh T, Tatsuta T, Sugawara S, Hara A and Hosono M: Synergistic anti-tumor effect of bullfrog sialic acid-binding lectin and pemetrexed in malignant mesothelioma. Oncotarget 8 42466-42477, 2017.

32. Altomare DA, Rybak SM, Pei J, Maizel JV, Cheung M, Testa JR and Shogen K: Onconase responsive genes in human mesothelioma cells: Implications for an RNA damaging therapeutic agent. BMC Cancer 10: 34, 2010.

33. Vert A, Castro J, Ribó M, Benito A and Vilanova M: Activating transcription factor 3 is crucial for antitumor activity and to strengthen the antiviral properties of Onconase. Oncotarget 8: 11692-11707, 2017.

34. Vert A, Castro J, Ribó M, Benito A and Vilanova M: A nuclear-directed human pancreatic ribonuclease (PE5) targets the metabolic phenotype of cancer cells. Oncotarget 7: 18309-18324, 2016.

35. Bolstad BM, Irizarry RA, Astrand M and Speed TP: A comparison of normalization methods for high density oligonucleotide array data based on variance and bias. Bioinformatics 19 185-193, 2003

36. Gentleman R, Carey V, Bates D, Bolstad B, Dettling M, Dudoit S Ellis B, Gautier L, Ge Y, Gentry J, et al: Bioconductor: Open software development for computational biology and bioinformatics. Genome Biol 5: R80, 2004.

37. Dudoit S, Gentleman RC and Quackenbush J: Open source software for the analysis of microarray data. Biotechniques 34: 496-501,2003.

38. Edgar R, Domrachev $M$ and Lash AE: Gene Expression Omnibus: NCBI gene expression and hybridization array data repository. Nucleic Acids Res 30: 207-210, 2002.

39. Ashburner M, Ball CA, Blake JA, Botstein D, Butler $\mathrm{H}$, Cherry JM, Davis AP, Dolinski K, Dwight SS, Eppig JT, et al: Gene ontology: Tool for the unification of biology. The Gene Ontology Consortium. Nat Genet 25: 25-29, 2000.

40. The Gene Ontology Consortium: The Gene Ontology Resource: 20 years and still GOing strong. Nucleic Acids Res 47: D330-D338, 2019

41. Kanehisa M: KEGG: Kyoto encyclopedia of genes and genomes Nucleic Acids Res 28: 27-30, 2000.

42. Dennis G Jr, Sherman BT, Hosack DA, Yang J, Gao W, Lane HC and Lempicki RA: DAVID: Database for annotation, visualization, and integrated discovery. Genome Biol 4: R60, 2003.

43. Warde-Farley D, Donaldson SL, Comes O, Zuberi K, Badrawi R, Chao P, Franz M, Grouios C, Kazi F, Lopes CT, et al: The GeneMANIA prediction server: Biological network integration for gene prioritization and predicting gene function. Nucleic Acids Res 38 (Web Server issue): W214-W220, 2010.
44. Balendiran GK, Martin HJ, El-Hawari Y and Maser E: Cancer biomarker AKR1B10 and carbonyl metabolism. Chem Biol Interact 178: 134-137, 2009.

45. Thuren T: Hepatic lipase and HDL metabolism. Curr Opin Lipidol 11: 277-283, 2000

46. Takashima S, Tsuji S and Tsujimoto M: Characterization of the second type of human beta-galactoside alpha 2,6-sialyltransferase (ST6Gal II), which sialylates Gal $\beta 1,4 \mathrm{GlcNAc}$ structures on oligosaccharides preferentially: Genomic analysis of human sialyltransferase genes. J Biol Chem 277: 45719-45728, 2002.

47. Jez JM, Flynn TG and Penning TM: A nomenclature system for the aldo-keto reductase superfamily. Adv Exp Med Biol 414: 579-589, 1997.

48. Barski OA, Tipparaju SM and Bhatnagar A: The aldo-keto reductase superfamily and its role in drug metabolism and detoxification. Drug Metab Rev 40: 553-624, 2008.

49. Mindnich RD and Penning TM: Aldo-keto reductase (AKR) superfamily: Genomics and annotation. Hum Genomics 3: 362-370, 2009

50. Fukumoto SI, Yamauchi N, Moriguchi H, Hippo Y, Watanabe A, Shibahara J, Taniguchi $\mathrm{H}$, Ishikawa $\mathrm{S}$, Ito $\mathrm{H}$, Yamamoto S, et al: Overexpression of the aldo-keto reductase family protein AKR1B10 is highly correlated with smokers non-small cell lung carcinomas. Clin Cancer Res 11: 1776-1785, 2005.

51. Cao D, Fan ST and Chung SSM: Identification and characterization of a novel human aldose reductase- like gene. J Biol Chem 273: 11429-11435, 1998.

52. Distefano JK and Davis B: Diagnostic and prognostic potential of akr1b10 in human hepatocellular carcinoma. Cancers (Basel) 11: 486, 2019.

53. Chung YT, Matkowskyj KA, Li H, Bai H, Zhang W, Tsao MS, Liao J and Yang GY: Overexpression and oncogenic function of aldo-keto reductase family 1B10 (AKR1B10) in pancreatic carcinoma. Mod Pathol 25: 758-766, 2012.

54. Fang CY, Lin YH and Chen CL: Overexpression of AKR1B10 predicts tumor recurrence and short survival in oral squamous cell carcinoma patients. J Oral Pathol Med 48: 712-719, 2019.

55. Huang P, Chandra V and Rastinejad F: Retinoic acid actions through mammalian nuclear receptors. Chem Rev 114: 233-254, 2014.

56. Huang L, He R, Luo W, Zhu YS, Li J, Tan T, Zhang X, Hu Z and Luo D: Aldo-Keto reductase family 1 member B10 inhibitors: Potential drugs for cancer treatment. Recent Pat Anticancer Drug Discov 11: 184-196, 2016

57. Ruiz FX, Porté S, Parés X and Farrés J: Biological role of aldo-keto reductases in retinoic acid biosynthesis and signaling. Front Pharmacol 3: 58, 2012.

58. Wang C, Yan R, Luo D, Watabe K, Liao DF and Cao D: Aldo-keto reductase family 1 member B10 promotes cell survival by regulating lipid synthesis and eliminating carbonyls. J Biol Chem 284: 26742-26748, 2009.

59. Shen $\mathrm{Y}$, Zhong L, Johnson $\mathrm{S}$ and Cao D: Human aldo-keto reductases 1B1 and 1B10: A comparative study on their enzyme activity toward electrophilic carbonyl compounds. Chem Biol Interact 191: 192-198, 2011.

60. Matsunaga T, Suzuki A, Kezuka C, Okumura N, Iguchi K, Inoue I, Soda M, Endo S, El-Kabbani O, Hara A and Ikari A: Aldo-keto reductase 1B10 promotes development of cisplatin resistance in gastrointestinal cancer cells through down-regulating peroxisome proliferator-activated receptor- $\gamma$-dependent mechanism. Chem Biol Interact 256: 142-153, 2016.

61. Matsunaga T, Yamane Y, Iida K, Endo S, Banno Y, El-Kabbani O and Hara A: Involvement of the aldo-keto reductase, AKR1B10, in mitomycin-c resistance through reactive oxygen species-dependent mechanisms. Anticancer Drugs 22: 402-408, 2011.

62. Zhong L, Shen H, Huang C, Jing H and Cao D: AKR1B10 induces cell resistance to daunorubicin and idarubicin by reducing $\mathrm{C} 13$ ketonic group. Toxicol Appl Pharmacol 255: 40-47, 2011.

63. Mundt F, Johansson HJ, Forshed J, Arslan S, Metintas M, Dobra K, Lehtiö J and Hjerpe A: Proteome screening of pleural effusions identifies galectin 1 as a diagnostic biomarker and highlights several prognostic biomarkers for malignant mesothelioma. Mol Cell Proteomics 13: 701-715, 2014.

64. Usami N, Fukui T, Kondo M, Taniguchi T, Yokoyama T, Mori S, Yokoi K, Horio Y, Shimokata K, Sekido Y and Hida T: Establishment and characterization of four malignant pleural mesothelioma cell lines from Japanese patients. Cancer Sci 97: 387-394, 2006 
65. Suzuki Y, Murakami H, Kawaguchi K, Tanigushi T, Fujii M, Shinjo K, Kondo Y, Osada H, Shimokata K, Horio Y, et al: Activation of the PI3K-AKT pathway in human malignant mesothelioma cells. Mol Med Rep 2: 181-188, 2009.

66. Endo S, Xia S, Suyama M, Morikawa Y, Oguri H, Hu D, Ao Y, Takahara S, Horino Y, Hayakawa Y, et al: Synthesis of potent and selective inhibitors of Aldo-Keto reductase 1B10 and their efficacy against proliferation, metastasis, and cisplatin resistance of lung cancer cells. J Med Chem 60: 8441-8455, 2017.

67. Plebuch M, Soldan M, Hungerer C, Koch L and Maser E: Increased resistance of tumor cells to daunorubicin after transfection of cDNAs coding for anthracycline inactivating enzymes. Cancer Lett 255: 49-56, 2007.

68. Shiiba M, Yamagami H, Yamamoto A, Minakawa Y, Okamoto A, Kasamatsu A, Sakamoto Y, Uzawa K, Takiguchi Y and Tanzawa H: Mefenamic acid enhances anticancer drug sensitivity via inhibition of aldo-keto reductase $1 \mathrm{C}$ enzyme activity. Oncol Rep 37: 2025-2032, 2017.

69. Vasiliou V, Vasiliou K and Nebert DW: Human ATP-binding cassette (ABC) transporter family. Hum Genomics 3: 281-290, 2009.
70. Pasello M, Giudice AM and Scotlandi K: The ABC subfamily A transporters: Multifaceted players with incipient potentialities in cancer. Semin Cancer Biol 60: 57-71, 2020.

71. Iwasaki H, Okabe T, Takara K, Yoshida Y, Hanashiro K and Oku H: Down-regulation of lipids transporter ABCA1 increases the cytotoxicity of nitidine. Cancer Chemother Pharmacol 66: 953-959, 2010.

72. Hudson AL, Weir C, Moon E, Harvie R, Klebe S, Clarke SJ, Pavlakis $\mathrm{N}$ and Howell VM: Establishing a panel of chemoresistant mesothelioma models for investigating chemo-resistance and identifying new treatments for mesothelioma. Sci Rep 4: 6152, 2014.

73. Milosevic V, Kopecka J, Salaroglio IC, Libener R, Napoli F, Izzo S, Orecchia S, Ananthanarayanan P, Bironzo P, Grosso F, et al: Wnt/IL-1//IL-8 autocrine circuitries control chemoresistance in mesothelioma initiating cells by inducing ABCB5. Int J Cancer 146: 192-207, 2020.

(i) (9) $(9)$ This work is licensed under a Creative Commons EY NG ND Attribution-NonCommercial-NoDerivatives 4.0 International (CC BY-NC-ND 4.0) License. 